doi: $10.13108 / 2020-12-1-103$

\title{
INTEGRATION OF EQUATIONS OF KAUP SYSTEM KIND WITH SELF-CONSISTENT SOURCE IN CLASS OF PERIODIC FUNCTIONS
}

\author{
A.B. YAKHSHIMURATOV, B.A. BABAJANOV
}

\begin{abstract}
In this paper, we consider the equations of Kaup system kind with a selfconsistent source in the class of periodic functions. We discuss the complete integrability of the considered nonlinear system of equations, which is based on the transformation to the spectral data of an associated quadratic pencil of Sturm-Liouville equations with periodic coefficients. In particular, Dubrovin-type equations are derived for the time-evolution of the spectral data corresponding to the solutions of equations of Kaup system kind with self-consistent source in the class of periodic functions. Moreover, it is shown that spectrum of the quadratic pencil of Sturm-Liouville equations with periodic coefficients associated with considering nonlinear system does not depend on time. In a one-gap case, we write the explicit formulae for solutions of the problem under consideration expressed in terms of the Jacobi elliptic functions. We show that if $p_{0}(x)$ and $q_{0}(x)$ are real analytical functions, the lengths of the gaps corresponding to these coefficients decrease exponentially. The gaps corresponding to the coefficients $p(x, t)$ and $q(x, t)$ are same. This implies that the solutions of considered problem $p(x, t)$ and $q(x, t)$ are real analytical functions in $x$.
\end{abstract}

Keywords: equations of Kaup system kind, quadratic pencil of Sturm-Liouville equations, inverse spectral problem, trace formulas, periodical potential.

Mathematics Subject Classification: 39A23, 35Q51, 34K13, 34K29

\section{INTRODUCTION}

In [1], D.J. Kaup proved that the nonlinear system of equations

$$
\left\{\begin{array}{l}
\eta_{\tau}=\Phi_{x x}+\beta^{2} \Phi_{x x x x}-\varepsilon \cdot\left(\Phi_{x} \eta\right)_{x} \\
\eta=\Phi_{\tau}+\frac{1}{2} \varepsilon \cdot \Phi_{x}^{2}
\end{array}\right.
$$

is completely integrable. The system describes the waves propagation in a shallow water. In [2], complex finite-gap multiphase solutions expressed in terms of the Riemann theta functions were considered. Multi-soliton solutions were found and the asymptotic behavior of these solutions was studied. In [3], [4] and [5], a real finite-gap regular solutions of Kaup system were studied. In [6], the 'Inverse Scattering Transform' was employed to solve a class of nonlinear equations associated with the inverse problem for the one-dimensional Schrodinger equation with the energy-dependent potential.

It is not difficult (see [2]) to confirm that after the transformations

$$
\eta=\frac{4 \beta^{2}}{\varepsilon}\left(q+p^{2}\right)+\frac{1}{\varepsilon},
$$

A.B. Yakhshimuratov, B.A. Babajanov, Integration of Equations of Kaup system kind with SELF-CONSISTENT SOURCE IN CLASS OF PERIODIC FUNCTIONS.

(c) A.B.Yakhshimuratov, B.A.Babajanov 2020.

Поступила 25 февраля 2019 г. 


$$
\begin{aligned}
& \Phi_{\tau}=\frac{4 \beta^{2}}{\varepsilon}\left(q+3 p^{2}\right)+\frac{1}{\varepsilon} \\
& \Phi_{x}=-\frac{4 \beta}{i \varepsilon} p, \quad t=i \beta \tau,
\end{aligned}
$$

the system of Kaup equations casts into a simpler form

$$
\left\{\begin{array}{l}
p_{t}=-6 p p_{x}-q_{x} \\
q_{t}=p_{x x x}-4 q p_{x}-2 p q_{x} .
\end{array}\right.
$$

We shall also call it the Kaup system.

The Kaup system can be considered as a compatibility condition (see [2])

$$
y_{x x t}-y_{t x x} \equiv\left[\left(q_{t}-p_{x x x}+4 q p_{x}+2 p q_{x}\right)+2 \lambda\left(p_{t}+6 p p_{x}+q_{x}\right)\right] y=0
$$

for the system of the linear equations

$$
\left\{\begin{array}{l}
-y_{x x}+q y+2 \lambda p y-\lambda^{2} y=0 \\
y_{t}+2 \lambda y_{x}+2 p y_{x}-p_{x} y=0
\end{array}\right.
$$

The first of these equations is called the quadratic pencil of Sturm-Liouville equations.

In [7], the Kaup system with self-consistent sources was studied by means of the inverse problem for the quadratic pencil of Sturm-Liouville equations with periodic potential. The inverse problem for the quadratic pencil of Sturm-Liouville equations with periodical potential on a half-line and the entire line was solved in the works [8]-[13].

Recently, nonlinear evolution equations with self-consistent sources have received much attention in the scientific literature. Physically, the sources appear in solitary waves with nonconstant velocity and lead to a variety of dynamics in physical models. They have important applications in plasma physics, hydrodynamics, solid-state physics, etc. [14]-[19]. For example, the $\mathrm{KdV}$ equation, which is included an integral type self-consistent source, was considered in [17]. This type equation can be employed to describe the interaction of long and short capillary-gravity waves [18]. Other important soliton equations with self-consistent source are the nonlinear Schrodinger equation, which describes the nonlinear interaction of an ion acoustic wave in the two component homogeneous plasma with the electrostatic high frequency wave [19]. Other aspects on integration of nonlinear systems were presented in [20]-[25].

In this paper, the method of the inverse spectral problem for the quadratic pencil of SturmLiouville equations with periodic coefficients is used to integrate the equation of Kaup system kind with a self-consistent source in the class of periodic functions. In the one-gap case, we write the explicit formulae for solutions of the problem under consideration, expressed in terms of the Jacobi elliptic functions.

The paper is organized as follows. In section 2, we present the formulation of the considered problem and we provide some basic information about the spectral theory of the quadratic pencil of Sturm-Liouville equations with periodic coefficients. Section 3 is devoted to describing the evolution of the spectral data corresponding to the problem in question. In section 4, we illustrate the application of the main result for the one-gap case. 


\section{Formulation OF PROBLEM}

We consider the system of equations with a self-consistent source

$$
\left\{\begin{aligned}
p_{t}= & p_{x x x}-6 p_{x} q-6 p q_{x}-30 p^{2} p_{x}+\sum_{k=-\infty}^{\infty} \alpha_{k}(t) s\left(\pi, \lambda_{k}, t\right)\left(\psi_{+}^{2}\left(x, \lambda_{k}, t\right)\right)_{x} \\
q_{t}= & q_{x x x}+6 p p_{x x x}+18 p_{x} p_{x x}-6 q q_{x}-24 p q p_{x}-6 p^{2} q_{x} \\
& +2 \sum_{k=-\infty}^{\infty} \alpha_{k}(t) s\left(\pi, \lambda_{k}, t\right)\left(-p_{x} \cdot \psi_{+}^{2}\left(x, \lambda_{k}, t\right)+\left(\lambda_{k}-2 p\right)\left(\psi_{+}^{2}\left(x, \lambda_{k}, t\right)\right)_{x}\right)
\end{aligned}\right.
$$

in the class of real-valued $\pi$-periodic in the spatial variable $x$ functions $p=p(x, t)$ and $q=q(x, t)$ possessing the following regularity

$$
p(x, t), q(x, t) \in C_{x}^{3}(t \geqslant 0) \cap C_{t}^{1}(t>0) \cap C(t \geqslant 0)
$$

and satisfying the initial conditions

$$
\left.p(x, t)\right|_{t=0}=p_{0}(x),\left.\quad q(x, t)\right|_{t=0}=q_{0}(x) .
$$

Here $p_{0}(x)$ and $q_{0}(x)$ are the given real-valued $\pi$-periodic functions such that for each nontrivial function $y(x) \in W_{2}^{2}[0, \pi]$ satisfying the identities

$$
y^{\prime}(0) \bar{y}(0)-y^{\prime}(\pi) \bar{y}(\pi)=0, \quad(y, y)=1,
$$

the following inequality holds:

$$
\left(p_{0} y, y\right)^{2}+\left(q_{0} y, y\right)+\left(y^{\prime}, y^{\prime}\right)>0
$$

where $(\cdot, \cdot)$ is a scalar product of the space $L_{2}(0, \pi)$. We observe that for $p(x)=0$ the equation (2.1) reduces to the Korteweg-de Vriez equation with a self-consistent source. In system (2.1), $\alpha_{k}(t), k \in Z$ is a given sequences of real-valued continuous functions having a uniform asymptotic behavior

$$
\alpha_{k}=O\left(\frac{1}{k^{3}}\right), \quad k \rightarrow \pm \infty
$$

and $\psi_{+}\left(x, \lambda_{k}, t\right)$ is the Floquet solution normalized by the condition $\psi_{+}\left(0, \lambda_{k}, t\right)=1$ of the quadratic pencil of Sturm-Liouville equations

$$
T(\lambda, t) y \equiv-y^{\prime \prime}+q y+2 \lambda p y-\lambda^{2} y=0, \quad x \in \mathbb{R} .
$$

Here $\lambda_{k}$ are the zeroes of the function $\Delta^{2}(\lambda)-4$, where $\Delta(\lambda)=c(\pi, \lambda, t)+s^{\prime}(\pi, \lambda, t)$. We denote by $c(x, \lambda, t)$ and $s(x, \lambda, t)$ the solutions of equation 2.3$)$ satisfying the initial conditions

$$
c(0, \lambda, t)=1, \quad c^{\prime}(0, \lambda, t)=0, \quad s(0, \lambda, t)=0, \quad s^{\prime}(0, \lambda, t)=1,
$$

respectively.

The spectrum of the quadratic pencil $(2.3)$ is real and coincides with the set [8-9]

$$
\sigma(T)=\{\lambda \in \mathbb{R} \mid-2 \leqslant \Delta(\lambda) \leqslant 2\}=\mathbb{R} \backslash \bigcup_{n=-\infty}^{\infty}\left(\lambda_{2 n-1}, \lambda_{2 n}\right) .
$$

The intervals $\left(\lambda_{2 n-1}, \lambda_{2 n}\right), n \in \mathbb{Z}$, are called the gaps or lacunas. The numbering is introduced in such a way that $\lambda_{-1}<0<\lambda_{0}$.

We denote by $\xi_{n}(t), n \in \mathbb{Z} \backslash\{0\}$ the eigenvalues of the Dirichlet problem $(y(0)=y(\pi)=0)$ for equation (2.3). The inclusions $\xi_{n}(t) \in\left[\lambda_{2 n-1}, \lambda_{2 n}\right]$ and the identity

$$
s(\pi, \lambda, t)=\pi \prod_{0 \neq k=-\infty}^{\infty} \frac{\xi_{k}(t)-\lambda}{k}
$$

are satisfied. 
The numbers $\xi_{n}=\xi_{n}(t)$ with the signs

$$
\sigma_{n}=\sigma_{n}(t)=\operatorname{sign}\left\{s^{\prime}\left(\pi, \xi_{n}\right)-c\left(\pi, \xi_{n}\right)\right\}, \quad n \in \mathbb{Z} \backslash\{0\},
$$

are called the spectral parameters of quadratic pencil $(2.3)$.

The boundaries $\lambda_{n}$ of the spectrum and the spectral parameters $\xi_{n}, \sigma_{n}$ are called the spectral data of problem $(2.3)$.

The aim of this work is to develop a procedure for constructing the solution $(p(x, t), q(x, t)$, $\left.\psi_{+}\left(x, \lambda_{k}, t\right)\right)$ of problem (2.1)-(2.3) by means of the inverse spectral problem for the quadratic pencil of Sturm-Liouville equations (2.3).

\section{MAIN RESULT}

The main result of the paper is presented in the following theorem.

Theorem 3.1. Let $p(x, t), q(x, t)$ and $\psi_{+}\left(x, \lambda_{k}, t\right)$ be solution of problem (2.1)- (2.3). Then the spectrum of problem (2.3) is independent of $t$, and the spectral parameters $\xi_{n}(t), n \in \mathbb{Z} \backslash\{0\}$ satisfy the following analogue of the system of Dubrovin equations

$$
\begin{aligned}
\dot{\xi}_{n}(t)= & 2(-1)^{n} \sigma_{n}(t) \operatorname{sign}(n) \cdot \sqrt{\left(\xi_{n}(t)-\lambda_{2 n-1}\right)\left(\lambda_{2 n}-\xi_{n}(t)\right)} \\
& \cdot \sqrt{\left(\xi_{n}(t)-\lambda_{-1}\right)\left(\xi_{n}(t)-\lambda_{0}\right) \prod_{k \neq n, 0} \frac{\left(\xi_{n}(t)-\lambda_{2 k-1}\right)\left(\xi_{n}(t)-\lambda_{2 k}\right)}{\left(\xi_{n}(t)-\xi_{k}(t)\right)^{2}}} \\
& \cdot\left(\left(4 \xi_{n}(t)+2\left(\lambda_{-1}+\lambda_{0}\right)+4 \sum_{0 \neq k=-\infty}^{\infty}\left(\frac{\lambda_{2 k-1}+\lambda_{2 k}}{2}-\xi_{k}(t)\right)\right) \xi_{n}(t)\right. \\
& +2\left(\frac{\lambda_{-1}+\lambda_{0}}{2}+\sum_{0 \neq k=-\infty}^{\infty}\left(\frac{\lambda_{2 k-1}+\lambda_{2 k}}{2}-\xi_{k}(t)\right)\right)^{2}+\lambda_{-1}^{2}+\lambda_{0}^{2} \\
& \left.+\sum_{0 \neq k=-\infty}^{\infty}\left(\lambda_{2 k-1}^{2}+\lambda_{2 k}^{2}-2 \xi_{k}^{2}(t)\right)+\sum_{k=-\infty}^{\infty} \frac{\alpha_{k}(t) s\left(\pi, \lambda_{k}, t, \tau\right)}{\xi_{n}(t)-\lambda_{k}}\right) .
\end{aligned}
$$

The sign $\sigma_{n}(t)= \pm 1$ changes at each collision of the point $\xi_{n}(t)$ with the boundaries of its gap $\left[\lambda_{2 n-1}, \lambda_{2 n}\right]$. Moreover, the following initial conditions are satisfied

$$
\left.\xi_{n}(t)\right|_{t=0}=\xi_{n}^{0},\left.\quad \sigma_{n}(t)\right|_{t=0}=\sigma_{n}^{0}, \quad n \in \mathbb{Z} \backslash\{0\},
$$

where $\xi_{n}^{0}, \sigma_{n}^{0}, n \in \mathbb{Z} \backslash\{0\}$ are the spectral parameters of the quadratic pencil of Sturm-Liouville equations corresponding to the coefficients $p_{0}(x)$ and $q_{0}(x)$.

Proof. Let $y_{n}(x, t)$ be the normalized eigenfunction of the Dirichlet problem for equation (2.3) corresponding to the eigenvalue $\xi_{n}=\xi_{n}(t)$. It is easy to see that

$$
y_{n}(x, t)=\frac{1}{c_{n}(t)} s\left(x, \xi_{n}(t), t\right),
$$

where

$$
c_{n}^{2}(t)=\int_{0}^{\pi} s^{2}\left(x, \xi_{n}(t), t\right) d x .
$$

Differentiating the identity

$$
-\left(y_{n}^{\prime \prime}, y_{n}\right)+\left(q y_{n}, y_{n}\right)+2 \xi_{n}\left(p y_{n}, y_{n}\right)-\xi_{n}^{2}=0,
$$

with respect to $t$, we get

$$
-\left(\dot{y}_{n}^{\prime \prime}, y_{n}\right)-\left(y_{n}^{\prime \prime}, \dot{y}_{n}\right)+\left(q_{t} y_{n}+q \dot{y}_{n}, y_{n}\right)+\left(q y_{n}, \dot{y}_{n}\right)+2 \dot{\xi}_{n}\left(p y_{n}, y_{n}\right)
$$




$$
+2 \xi_{n}\left(p_{t} y_{n}+p \dot{y}_{n}, y_{n}\right)+2 \xi_{n}\left(p y_{n}, \dot{y}_{n}\right)-2 \xi_{n} \dot{\xi}_{n}=0
$$

By the last identity we obtain

$$
\begin{aligned}
& \left(-\dot{y}_{n}^{\prime \prime}+q \dot{y}_{n}+2 \xi_{n} p \dot{y}_{n}, y_{n}\right)+\left(-y_{n}^{\prime \prime}+q y_{n}+2 \xi_{n} p y_{n}, \dot{y}_{n}\right) \\
& +\left(q_{t} y_{n}+2 \xi_{n} p_{t} y_{n}, y_{n}\right)+2 \dot{\xi}_{n}\left(p y_{n}, y_{n}\right)-2 \xi_{n} \dot{\xi}_{n}=0, \\
& 2 \dot{\xi}_{n}\left[\xi_{n}-\left(p y_{n}, y_{n}\right)\right]=\left(q_{t} y_{n}+2 \xi_{n} p_{t} y_{n}, y_{n}\right)
\end{aligned}
$$

and hence,

$$
2 \dot{\xi}_{n}\left(\xi_{n}-\int_{0}^{\pi} p y_{n}^{2} d x\right)=\int_{0}^{\pi}\left(q_{t}+2 \xi_{n} p_{t}\right) y_{n}^{2} d x
$$

According to (2.1) we have

$$
\begin{aligned}
q_{t}+2 \xi_{n} p_{t}= & q_{x x x}+6 p p_{x x x}+18 p_{x} p_{x x}-6 q q_{x}-24 p q p_{x}-6 p^{2} q_{x} \\
& +2 \xi_{n}\left(p_{x x x}-6 p_{x} q-6 p q_{x}-30 p^{2} p_{x}\right)+G_{2}+2 \xi_{n} G_{1}
\end{aligned}
$$

where

$$
G_{1}(x, t)=\sum_{k=-\infty}^{\infty} \alpha_{k}(t) s\left(\pi, \lambda_{k}, t\right)\left(\psi_{+}^{2}\left(x, \lambda_{k}, t\right)\right)_{x}
$$

and

$$
G_{2}(x, t)=2 \sum_{k=-\infty}^{\infty} \alpha_{k}(t) s\left(\pi, \lambda_{k}, t\right)\left\{-p_{x} \cdot \psi_{+}^{2}\left(x, \lambda_{k}, t\right)+\left(\lambda_{k}-2 p\right)\left(\psi_{+}^{2}\left(x, \lambda_{k}, t\right)\right)_{x}\right\}
$$

We introduce the polynomial

$$
c\left(x, t, \xi_{n}\right)=c_{0}(x, t) \xi_{n}^{2}+c_{1}(x, t) \xi_{n}+c_{2}(x, t)
$$

and we rewrite identity (3.5) as

$$
q_{t}+2 \xi_{n} p_{t}=\frac{1}{2} c^{\prime \prime \prime}+2 c^{\prime} \cdot\left(\xi_{n}^{2}-2 p \xi_{n}-q\right)-c \cdot\left(2 p^{\prime} \xi_{n}+q^{\prime}\right)+G_{2}+2 \xi_{n} G_{1}
$$

where

$$
c_{0}(x, t)=4, c_{1}(x, t)=4 p(x, t), c_{2}(x, t)=2\left[q(x, t)+3 p^{2}(x, t)\right] .
$$

Using the identity

$$
q y_{n}=\xi_{n}^{2} y_{n}+y_{n}^{\prime \prime}-2 \xi_{n} p y_{n}
$$

it is easy to show that

$$
\begin{aligned}
& \left(\left(\frac{1}{2} c^{\prime \prime}+c \cdot\left(\xi_{n}^{2}-2 p \xi_{n}-q\right)\right) y_{n}^{2}-c^{\prime} y_{n} y_{n}^{\prime}+c\left(y_{n}^{2}\right)^{\prime}\right)^{\prime} \\
& =\left(\frac{1}{2} c^{\prime \prime \prime}+2 c^{\prime} \cdot\left(\xi_{n}^{2}-2 p \xi_{n}-q\right)-c \cdot\left(2 p^{\prime} \xi_{n}+q^{\prime}\right)\right) y_{n}^{2}
\end{aligned}
$$


Substituting expressions (3.6) into formula (3.4) and taking into consideration (3.7), we arrive at the identity

$$
\begin{aligned}
2 \dot{\xi}_{n}\left(\xi_{n}-\int_{0}^{\pi} p y_{n}^{2} d x\right)= & \left.\left(\left(\frac{1}{2} c^{\prime \prime}+c \cdot\left(\xi_{n}^{2}-2 p \xi_{n}-q\right)\right) y_{n}^{2}-c^{\prime} y_{n} y_{n}^{\prime}+c\left(y_{n}^{2}\right)^{\prime}\right)\right|_{0} ^{\pi} \\
& +\int_{0}^{\pi}\left(G_{2}+2 \xi_{n} G_{1}\right) y_{n}^{2} d x \\
= & c\left(0, t, \xi_{n}\right)\left(y_{n}^{\prime 2}(\pi, t)-y_{n}^{\prime 2}(0, t)\right)+\int_{0}^{\pi}\left(G_{2}+2 \xi_{n} G_{1}\right) y_{n}^{2} d x .
\end{aligned}
$$

Now we calculate the last integral in 3.8

$$
\begin{aligned}
I \equiv & \int_{0}^{\pi}\left(G_{2}+2 \xi_{n} G_{1}\right) y_{n}^{2} d x \\
= & \sum_{k=-\infty}^{\infty} \alpha_{k} s\left(\pi, \lambda_{k}, t\right) \\
& \quad \cdot \int_{0}^{\pi}\left(-2 p_{x} y_{n}^{2} \cdot \psi_{+}^{2}\left(x, \lambda_{k}, t\right)+2\left(\xi_{n}+\lambda_{k}-2 p\right) y_{n}^{2} \cdot\left(\psi_{+}^{2}\left(x, \lambda_{k}, t\right)\right)_{x}\right) d x
\end{aligned}
$$

It is easy to confirm that

$$
\begin{aligned}
J \equiv & -2 \int_{0}^{\pi} p_{x} y_{n}^{2} \psi_{k}^{2} d x+2 \int_{0}^{\pi}\left(\xi_{n}+\lambda_{k}-2 p\right) y_{n}^{2}\left(\psi_{k}^{2}\right)_{x} d x \\
= & -2 \int_{0}^{\pi} p_{x} y_{n}^{2} \psi_{k}^{2} d x+\int_{0}^{\pi}\left(\xi_{n}+\lambda_{k}-2 p\right) y_{n}^{2}\left(\psi_{k}^{2}\right)_{x} d x+\int_{0}^{\pi}\left(\xi_{n}+\lambda_{k}-2 p\right) y_{n}^{2} d\left(\psi_{k}^{2}\right) \\
= & -2 \int_{0}^{\pi} p_{x} y_{n}^{2} \psi_{k}^{2} d x+\int_{0}^{\pi} 2\left(\xi_{n}+\lambda_{k}-2 p\right) y_{n}^{2} \psi_{k} \psi_{k}^{\prime} d x \\
& -\int_{0}^{\pi}\left(-2 p_{x} y_{n}^{2}+2\left(\xi_{n}+\lambda_{k}-2 p\right) y_{n} y_{n}^{\prime}\right) \psi_{k}^{2} d x \\
= & \int_{0}^{\pi} 2\left(\xi_{n}+\lambda_{k}-2 p\right) y_{n} \psi_{k}\left(y_{n} \psi_{k}^{\prime}-y_{n}^{\prime} \psi_{k}\right) d x,
\end{aligned}
$$

where $\psi_{k}=\psi_{+}\left(x, \lambda_{k}, t\right)$. Using the identity

$$
\left(\xi_{n}+\lambda_{k}-2 p\right) y_{n} \psi_{k}=\frac{\left(y_{n} \psi_{k}^{\prime}-y_{n}^{\prime} \psi_{k}\right)^{\prime}}{\xi_{n}-\lambda_{k}}
$$

by 3.10 we obtain that

$$
J=\frac{1}{\xi_{n}-\lambda_{k}}\left(y_{n}^{\prime 2}(\pi, t)-y_{n}^{\prime 2}(0, t)\right) .
$$


Substituting (3.11) into (3.9), we get

$$
I=\sum_{k=-\infty}^{\infty} \frac{\alpha_{k}(t) s\left(\pi, \lambda_{k}, t\right)}{\xi_{n}-\lambda_{k}} \cdot\left(y_{n}^{\prime 2}(\pi, t)-y_{n}^{\prime 2}(0, t)\right) .
$$

Hence, by means of expression (3.12), we conclude that

$$
\begin{aligned}
& 2 \dot{\xi}_{n}\left(\xi_{n}-\int_{0}^{\pi} p y_{n}^{2} d x\right)=\left(y_{n}^{\prime 2}(\pi, t)-y_{n}^{\prime 2}(0, t)\right) \sum_{k=0}^{2} c_{k}(0, t) \xi_{n}^{2-k} \\
& \quad+\left(y_{n}^{\prime 2}(\pi, t)-y_{n}^{\prime 2}(0, t)\right) \sum_{k=-\infty}^{\infty} \frac{\alpha_{k}(t) s\left(\pi, \lambda_{k}, t\right)}{\xi_{n}-\lambda_{k}} .
\end{aligned}
$$

By virtue of (3.3), identity 3.13) can be written as

$$
\begin{aligned}
2 \dot{\xi}_{n}(t)\left(\xi_{n}(t) c_{n}^{2}(t)-\int_{0}^{\pi} p s^{2}\left(x, \xi_{n}(t), t\right) d x\right) & =\left(s^{\prime 2}\left(\pi, \xi_{n}(t), t\right)-1\right) \sum_{k=0}^{2} c_{k}(0, t) \xi_{n}^{2-k} \\
& +\left(s^{\prime 2}\left(\pi, \xi_{n}(t), t\right)-1\right) \sum_{k=-\infty}^{\infty} \frac{\alpha_{k}(t) s\left(\pi, \lambda_{k}, t\right)}{\xi_{n}-\lambda_{k}} .
\end{aligned}
$$

It follows from the identity

$$
2 \xi_{n}(t) c_{n}^{2}(t)-2 \int_{0}^{\pi} p(x, t) s^{2}\left(x, \xi_{n}(t), t\right) d x=s^{\prime}\left(\pi, \xi_{n}(t), t\right) \frac{\partial s\left(\pi, \xi_{n}(t), t\right)}{\partial \lambda}
$$

that

$$
\begin{aligned}
\dot{\xi}_{n}(t) \frac{\partial s\left(\pi, \xi_{n}(t), t\right)}{\partial \lambda}= & \left(s^{\prime}\left(\pi, \xi_{n}(t), t\right)-\frac{1}{s^{\prime}\left(\pi, \xi_{n}(t), t\right)}\right) \sum_{k=0}^{2} c_{k}(0, t) \xi_{n}^{2-k} \\
& +\left(s^{\prime}\left(\pi, \xi_{n}(t), t\right)-\frac{1}{s^{\prime}\left(\pi, \xi_{n}(t), t\right)}\right) \sum_{k=-\infty}^{\infty} \frac{\alpha_{k}(t) s\left(\pi, \lambda_{k}, t\right)}{\xi_{n}-\lambda_{k}} .
\end{aligned}
$$

Now, substituting the values $x=\pi$ and $\lambda=\xi_{n}(t)$ into the identity

$$
c(x, \lambda, t) s^{\prime}(x, \lambda, t)-c^{\prime}(x, \lambda, t) s(x, \lambda, t)=1,
$$

we find that

$$
c\left(\pi, \xi_{n}(t), t\right)=\frac{1}{s^{\prime}\left(\pi, \xi_{n}(t), t\right)} .
$$

By (3.15) and the identity

$$
\left[c(\pi, \lambda, t)-s^{\prime}(\pi, \lambda, t)\right]^{2}=\left(\Delta^{2}(\lambda)-4\right)-4 c^{\prime}(\pi, \lambda, t) s(\pi, \lambda, t),
$$

we arrive at the identity

$$
s^{\prime}\left(\pi, \xi_{n}(t), t\right)-\frac{1}{s^{\prime}\left(\pi, \xi_{n}(t), t\right)}=\sigma_{n}(t) \sqrt{\Delta^{2}\left(\xi_{n}(t)\right)-4} .
$$

Using (2.4), (3.16) and the expansion

$$
\Delta^{2}(\lambda)-4=-4 \pi^{2}\left(\lambda-\lambda_{-1}\right)\left(\lambda-\lambda_{0}\right) \prod_{0 \neq k=-\infty}^{\infty} \frac{\left(\lambda-\lambda_{2 k-1}\right)\left(\lambda-\lambda_{2 k}\right)}{k^{2}},
$$


we find:

$$
\begin{aligned}
\frac{s^{\prime}\left(\pi, \xi_{n}(t), t\right)-\frac{1}{s^{\prime}\left(x, \xi_{n}(t), t\right)}}{\frac{\partial s\left(\pi, \xi_{n}(t), t\right)}{\partial \lambda}} & =\frac{\sigma_{n}(t) \sqrt{\Delta^{2}\left(\xi_{n}(t)\right)-4}}{\frac{\partial s\left(\pi, \xi_{n}(t), t\right)}{\partial \lambda}} \\
& =2(-1)^{n} \sigma_{n}(t) \operatorname{sign}(n) \cdot \sqrt{\left(\xi_{n}(t)-\lambda_{2 n-1}\right)\left(\lambda_{2 n}-\xi_{n}(t)\right)} \\
& \cdot \sqrt{\left(\xi_{n}(t)-\lambda_{-1}\right)\left(\xi_{n}(t)-\lambda_{0}\right) \prod_{k \neq n, 0} \frac{\left(\xi_{n}(t)-\lambda_{2 k-1}\right)\left(\xi_{n}(t)-\lambda_{2 k}\right)}{\left(\xi_{n}(t)-\xi_{k}(t)\right)^{2}}} .
\end{aligned}
$$

Here we have also employed the identity

$$
\operatorname{sign}\left(-\frac{\pi}{n} \prod_{k \neq n, 0} \frac{\xi_{k}(t)-\xi_{n}(t)}{k}\right)=(-1)^{n} \operatorname{sign}(n) .
$$

From (3.14), (3.17) and the trace formulae

$$
\begin{aligned}
& p(t)=\frac{\lambda_{-1}+\lambda_{0}}{2}+\sum_{0 \neq k=-\infty}^{\infty}\left(\frac{\lambda_{2 k-1}+\lambda_{2 k}}{2}-\xi_{k}(t)\right), \\
& q(t)+2 p^{2}(t)=\frac{\lambda_{-1}^{2}+\lambda_{0}^{2}}{2}+\sum_{0 \neq k=-\infty}^{\infty}\left(\frac{\lambda_{2 k-1}^{2}+\lambda_{2 k}^{2}}{2}-\xi_{k}^{2}(t)\right)
\end{aligned}
$$

we get (3.1).

We note that if instead of the Dirichlet boundary conditions we consider periodic or antiperiodic boundary value conditions, then equation 3.13 becomes $\dot{\lambda}_{n}(t)=0, n \in Z$. Hence, the spectrum of problem $(2.3)$ is independent of the parameter $t$, and this completes the proof.

Remark 1. If instead of $p(x, t)$ and $q(x, t)$ we consider the functions $p(x+\tau, t)$ and $q(x+\tau, t)$, then, as we have seen in the previous section, the eigenvalues of the periodic and antiperiodic problems are independent of the parameters $\tau$ and $t$. However, the eigenvalues $\xi_{n}$ of the Dirichlet problem and the signs $\sigma_{n}$ do depend on $\tau$ and $t: \xi_{n}=\xi_{n}(\tau, t), \sigma_{n}=\sigma_{n}(\tau, t)= \pm 1$.

Remark 2. The theorem gives a method for solving problem (2.1)-(2.3). First we find the spectral data $\lambda_{n}, n \in Z, \xi_{n}^{0}(\tau), \sigma_{n}^{0}(\tau), n \in \mathbb{Z} \backslash\{0\}$ of the quadratic pencil of Sturm-Liouville equations corresponding to the coefficients $p_{0}(x+\tau)$ and $q_{0}(x+\tau)$. Then we solve the Cauchy problem for Dubrovin system (3.1) with the initial conditions

$$
\left.\xi_{n}(\tau, t)\right|_{t=0}=\xi_{n}^{0}(\tau),\left.\quad \sigma_{n}(\tau, t)\right|_{t=0}=\sigma_{n}^{0}(\tau), \quad n \in \mathbb{Z} \backslash\{0\} .
$$

Finally, by using the trace formulae

$$
\begin{gathered}
p(\tau, t)=\frac{\lambda_{-1}+\lambda_{0}}{2}+\sum_{0 \neq k=-\infty}^{\infty}\left(\frac{\lambda_{2 k-1}+\lambda_{2 k}}{2}-\xi_{k}(\tau, t)\right), \\
q(\tau, t)+2 p^{2}(\tau, t)=\frac{\lambda_{-1}^{2}+\lambda_{0}^{2}}{2}+\sum_{0 \neq k=-\infty}^{\infty}\left(\frac{\lambda_{2 k-1}^{2}+\lambda_{2 k}^{2}}{2}-\xi_{k}^{2}(\tau, t)\right)
\end{gathered}
$$

we get the expressions of $p(\tau, t)$ and $q(\tau, t)$. After that the Floquet solutions $\psi_{+}\left(x, \lambda_{k}, t\right)$ of equation (2.3) can be found easily.

Remark 3. If the number of zones is finite, that is, there are two nonnegative integer numbers $N$ and $M$ such that $\lambda_{2 k-1}=\lambda_{2 k}=\xi_{k}$ for all $k>N$ and $-M<k$, then system (3.1) reads as

$$
\frac{\partial \xi_{n}}{\partial t}=2(-1)^{n} \sigma_{n}(\tau, t) \operatorname{sign}(n) \cdot \sqrt{\left(\xi_{n}-\lambda_{2 n-1}\right)\left(\lambda_{2 n}-\xi_{n}\right)}
$$




$$
\begin{aligned}
& \sqrt{\left(\xi_{n}-\lambda_{-1}\right)\left(\xi_{n}-\lambda_{0}\right) \prod_{\substack{k=-M \\
k \neq n, 0}}^{N} \frac{\left(\xi_{n}-\lambda_{2 k-1}\right)\left(\xi_{n}-\lambda_{2 k}\right)}{\left(\xi_{n}-\xi_{k}\right)^{2}}} \\
& \cdot\left(\left(4 \xi_{n}+2\left(\lambda_{-1}+\lambda_{0}\right)+4 \sum_{0 \neq k=-M}^{N}\left(\frac{\lambda_{2 k-1}+\lambda_{2 k}}{2}-\xi_{k}(\tau, t)\right)\right) \xi_{n}\right. \\
& +2\left(\frac{\lambda_{-1}+\lambda_{0}}{2}+\sum_{0 \neq k=-M}^{N}\left(\frac{\lambda_{2 k-1}+\lambda_{2 k}}{2}-\xi_{k}(\tau, t)\right)\right)^{2}+\lambda_{-1}^{2}+\lambda_{0}^{2} \\
& \left.+\sum_{0 \neq k=-M}^{N}\left(\lambda_{2 k-1}^{2}+\lambda_{2 k}^{2}-2 \xi_{k}^{2}(\tau, t)\right)+\sum_{k=-M}^{N} \frac{\alpha_{k}(t) s\left(\pi, \lambda_{k}, t, \tau\right)}{\xi_{n}-\lambda_{k}}\right),
\end{aligned}
$$

where $n=-M, \ldots,-1,1, \ldots, N$.

Remark 4. In [13], there was proved the theorem stating that the lengths of the gaps of the quadratic pencil of Sturm-Liouvelle equations with $\pi$-periodic real-valued coefficients decrease exponentially if and only if the coefficients are analytic. From this theorem we conclude that if $p_{0}(x)$ and $q_{0}(x)$ are real analytical functions, then the lengths of the gaps corresponding to these coefficients decrease exponentially. The gaps corresponding to the coefficients $p(x, t)$ and $q(x, t)$ are same. Hence, the solutions $p(x, t)$ and $q(x, t)$ of problem (2.1)-(2.3) are real analytical functions on $x$.

Remark 5. In [26], an analogue of Borg inverse theorem was proved: the number $\frac{\pi}{2}$ is a period of the coefficients of the quadratic pencil of Sturm-Liouvelle equations with $\pi$-periodic real-valued coefficients if and only if all eigenvalues of antiperiodic problem are double. By this theorem we conclude that if the functions $p_{0}(x)$ and $q_{0}(x)$ have the period $\frac{\pi}{2}$, then all eigenvalues of antiperiodic problem corresponding to these coefficients are double. The gaps corresponding to the coefficients $p(x, t)$ and $q(x, t)$ are same. Hence, the solutions $p(x, t)$ and $q(x, t)$ of problem (2.1)-(2.3) are $\frac{\pi}{2}$-periodic functions in $x$.

\section{EXAMPLE}

We now illustrate the application of Theorem to solve the problem $(2.1)-(2.2)$.

Let us consider the following initial value conditions

$$
\begin{aligned}
\left.p(x, t)\right|_{t=0} & =p_{0}(x)=\frac{3-4 s n^{2}\left(3 x, \frac{2}{3}\right)}{1+2 c n^{2}\left(3 x, \frac{2}{3}\right)} \\
\left.q(x, t)\right|_{t=0} & =q_{0}(x)=\frac{81-156 s n^{2}\left(3 x, \frac{2}{3}\right)+72 s n^{4}\left(3 x, \frac{2}{3}\right)}{\left(1+2 c n^{2}\left(3 x, \frac{2}{3}\right)\right)^{2}},
\end{aligned}
$$

for equations of Kaup system kind 2.1 with $\alpha_{k}(t)=\frac{1}{\pi k^{3}}$. Let us find the spectral data of the problem (2.3) for $p_{0}(x)$ and $q_{0}(x)$. It has the form

$$
\lambda_{-1}=-1, \quad \lambda_{0}=1, \quad \lambda_{1}=2, \quad \lambda_{2}=4, \quad \xi_{1}^{0}(0)=2, \quad \sigma_{1}^{0}(0)=1 .
$$

In this case the system (3.1) reads as

$$
\frac{\partial \xi_{1}}{\partial t}=-226 \sigma_{n}(\tau, t) \cdot \sqrt{\left(4-\xi_{1}\right)\left(\xi_{1}-2\right)\left(\xi_{1}-1\right)\left(\xi_{1}+1\right)} .
$$

We consider this system subject to the initial condition

$$
\left.\xi_{1}(\tau, t)\right|_{t=0}=\xi_{1}^{0}(\tau),\left.\quad \sigma_{1}(\tau, t)\right|_{t=0}=\sigma_{1}^{0}(\tau),
$$


where $\xi_{1}^{0}(\tau)$ solves the differential equation

$$
\frac{d \xi_{1}^{0}(\tau)}{d \tau}=2 \sigma_{1}^{0}(\tau) \sqrt{\left(4-\xi_{1}^{0}(\tau)\right)\left(\xi_{1}^{0}(\tau)-2\right)\left(\xi_{1}^{0}(\tau)-1\right)\left(\xi_{1}^{0}(\tau)+1\right)}
$$

and satisfies the initial condition

$$
\left.\xi_{1}^{0}(\tau)\right|_{\tau=0}=2,\left.\quad \sigma_{1}^{0}(\tau)\right|_{\tau=0}=1 .
$$

Solving the Cauchy problem (4.1)- 4.2 we find that (see [27])

$$
\xi_{1}(\tau, t)=\frac{6-2 \mathrm{sn}^{2}\left(-339 t+3 \tau, \frac{2}{3}\right)}{1+2 \mathrm{cn}^{2}\left(-339 t+3 \tau, \frac{2}{3}\right)} .
$$

Substituting this into the first and second trace formulae, we obtain the solution of the given problem:

$$
\begin{gathered}
p(\tau, t)=\frac{3-4 \mathrm{sn}^{2}\left(-339 t+3 \tau, \frac{2}{3}\right)}{1+2 \mathrm{cn}^{2}\left(-339 t+3 \tau, \frac{2}{3}\right)} \\
q(\tau, t)=\frac{81-156 \mathrm{sn}^{2}\left(-339 t+3 \tau, \frac{2}{3}\right)+72 \mathrm{sn}^{4}\left(-339 t+3 \tau, \frac{2}{3}\right)}{\left(1+2 \mathrm{cn}^{2}\left(-339 t+3 \tau, \frac{2}{3}\right)\right)^{2}}
\end{gathered}
$$

where sn and cn are the Jacobi elliptic functions.

\section{ACKNOWLEDGEMENTS}

The authors express their gratitude to Prof. Aknazar Khasanov (Samarkand State University, Uzbekistan) for discussion and valuable advice, as well as to the International Erasmus+ Program KA106-2, Keele University, UK.

\section{BIBLIOGRAPHY}

1. D.J. Kaup. A higher-order water-wave equation and the method for solving it // Prog. Theor. Phys. 54:2, 396-408 (1975).

2. V.B. Matveev and M.I. Yavor. Solutions Presque périodiques et a n-solitons de l'équation hydrodynamique nonlinéaire de Kaup // Ann. Inst. Henri Poincare, Sect. A. 31:1, 25-41 (1979).

3. A.O. Smirnov. Real finite-gap regular solutions of the Kaup-Boussinesq equation // Theor. Math. Phys. 66:1, 19-31 (1986).

4. A.O. Smirnov. A matrix analogue of Appell's theorem and reductions of multi-dimensional Riemann theta-functions // Math. USSR-Sb. 61:2, 379-388 (1988).

5. Yu.A. Mitropol'skii, N.N. Bogolyubov (Jr.), A.K. Prikarpatskii and V.G. Samoilenko. Integrable dynamical systems: spectral and differential geometric aspects. Naukova Dumka, Kiev (1987). (in Russian).

6. M. Jaulent and I. Miodek. Nonlinear evolution equation associated with energy-dependent Schrödinger potentials // Lett. Math. Phys. 1:3, 243-250 (1976).

7. A. Cabada and A. Yakhshimuratov. The system of Kaup equations with a self-consistent source in the class of periodic functions // J. Math. Phys. Anal. Geom. 9:3, 287-303 (2013).

8. G.Sh. Guseinov. On a quadratic pencil of Sturm-Liouville operators with periodic coefficients // Vestnik Moskov. Univ., Ser. 1 Math. Mekh. 3:2, 14-21 (1984). (in Russian).

9. G.Sh. Guseinov. Spectrum and eigenfunction expansions of a quadratic pencil of Sturm-Liouville operators with periodic coefficients // in "Spectral theory of operators and its applications". 6, 56-97, "Elm", Baku (1985).

10. G.Sh. Guseinov. On Spectral analysis of a quadratic pencil of Sturm-Liouville operators // Soviet Math. Dokl. 32:3, 1859-1862 (1985).

11. G.Sh. Guseinov. Inverse problems for a quadratic pencil of Sturm-Liouville operators on a finite interval // in "Spectral theory of operators and its applications". 7, 51-101, "Elm", Baku (1986).

12. B.A. Babadzhanov, A.B. Khasanov. Inverse problem for a quadratic pencil of Shturm-Liouville operators with finite-gap pereodic potential on the half-line // Diff. Equats. 43:6, 723-730 (2007). 
13. B.A. Babadzhanov, A.B. Khasanov and A.B. Yakhshimuratov. On the inverse problem for a quadratic pencil of Sturm-Liouville operators with periodic potential // Diff. Equats. 41:3, 310$318(2005)$.

14. V.K. Melnikov. A direct method for deriving a multisoliton solution for the problem of interaction of waves on the x, y plane // Comm. Math. Phys. 112:4, 639-52 (1987).

15. V.K. Melnikov. Integration method of the Korteweg-de Vries equation with a self-consistent source // Phys. Lett. A. 133:9, 493-496 (1988).

16. V.K. Melnikov. Integration of the nonlinear Schrödinger equation with a self-consistent source // Comm. Math. Phys. 137:2, 359-381 (1991).

17. V.K. Melnikov. Integration of the Korteweg-de Vries equation with a source // Inverse Problems. 6:2, 233-246 (1990).

18. J. Leon, A. Latifi. Solution of an initial-boundary value problem for coupled nonlinear waves // J. Phys. A: Math. Gen. 23:8, 1385-1403 (1990).

19. C. Claude, J. Leon, A. Latifi. Nonlinear resonant scattering and plasma instability: an integrable model // J. Math. Phys. 32:12, 3321-3330 (1991).

20. V.S. Shchesnovich, E.V. Doktorov. Modified Manakov system with self-consistent source // Phys. Lett. A. 213:1-2, 23-31 (1996).

21. D.J. Zhang, D.Y. Chen. The $N$-soliton solutions of the sine-Gordon equation with self-consistent sources // Physica A: Stat. Mech. Appl. 321:3-4, 467-481 (2003).

22. P.G. Grinevich and I.A. Taimanov. Spectral conservation laws for periodic nonlinear equations of the Melnikov type // in "Geometry, topology, and mathematical physics. S. P. Novikov's seminar: 2006-2007", V.M. Buchstaber, I.M. Krichever (eds.) Amer. Math. Soc. Transl. Ser. 2. 224, 125-138 (2008).

23. A.B. Khasanov and A.B. Yakhshimuratov. The Korteweg-de Vries equation with a self-consistent source in the class of periodic functions // Theor. Math. Phys. 164:2, 1008-1015 (2010).

24. A.B. Yakhshimuratov. The nonlinear Schrödinger equation with a self-consistent source in the class of periodic functions // Math. Phys. Anal. Geom. 14:2, 153-169 (2011).

25. R. Yamilov. Symmetries as integrability criteria for differential difference equations // J. Phys. A: Math. Gen. 39:45, R541-R623 (2006).

26. A.B. Yakhshimuratov. Analogue of the inverse theorem of Borg for a quadratic pencil of operators of Sturm-Liouville // Bull. Eletski State University. Ser. Math. Comp. Math. 8:1, 121-126 (2005).

27. I.S. Gradshteyn, I.M. Ryzhik. Table of integrals, series, and products. Fiz.-Matem. Liter., Moscow (1963). [Academic Press, San Diego (2000).]

Alisher Bekchanovich Yakhshimuratov,

Urgench Branch of Tashkent University

of Information Technologies

named after Muhammad al-Khwarizmi,

Al-Khwarizmi street, 110,

220100, Urgench city, Uzbekistan

E-mail: albaron@mail.ru

Bazar Atajanovich Babajanov,

Urgench State University,

Hamid Alimjan street, 14,

220100, Urgench city, Uzbekistan

E-mail: a.murod@mail.ru 